\title{
Subsídios para o estudo das Lecythidaceae da Amazônia
}

\author{
WILliam A. RoDRigues (") \\ Instituto Nacional de Pesquisas \\ da Amazônia
}

\begin{abstract}
SINOPSE
As Lecitidáceas Corythophora rimosa W. Rodr., Cariniana pauciramosa W. Rodr., Couratari longipedicellata W. Rodr. e Couratari prancei W. Rodr. são descritas cumo novas e Corythophora alta $R$. Knuth, Couratari multiflora (J. E. Smith) Eyma, C. stellata A. C. Smith, C. oligantha Sandwith e C. duckei $\mathrm{R}$. Knuth são discutidas como espécies pouco conhecidas da Amazônia.
\end{abstract}

\section{INTRODUÇÃo}

As Lecitidáceas constituem uma das familias mais marcantes da flora amazônica não só pela abundância de exemplares como pela importância econômica que representam algumas de suas espécies. Apesar de sua importância, há muito está carecendo de uma revisão taxonômica. Embora o estudo de alguns de seus gêneros năo represente problema, o mesmo não se pode dizer por exemplo com Lecythis e Eschweilera, gêneros complexos e, por não dizer, confusos com muitas espécies mal e deficientemente descritas e muitos tipos importantes irremediavelmente perdidos durante a última guerra mundial.

\section{1 - Corythophora Knuth}

1 Corythophora rimosa W. Rodrigues, n. sp.

(Fig. 1)

Arbor ad $30 \mathrm{~m}$ alta, radicibus tabularibus destituta, trunco cylindrico cortice profunde fisso vestito; ramuli juniores glabri squamulosique. Foliorum lamina coriacea, elliptica, $4,0-16,0 \mathrm{~cm}$ longa, $2,0-9,0 \mathrm{~cm}$ lata, utrimque glabra, ad apicem acuminata, acumine $1,0-10,0$ $\mathrm{mm}$ longo, ad basin rotundata vel breviter angustata, ad marginem undulata vel serrulatoundulata; costa supra prominula, subtus pro minens; nervi laterales utrimque 12-18, brochidodromi, supra prominuli, subtus prominentes; rete venularum utrimque prominulum; petiolus $4,0-13,0 \mathrm{~mm}$ longus, $1,0-(2,0)-3,0 \mathrm{~mm}$ latus, canaliculatus, nigrescens, glaber. Inflorescentia terminalis et subterminalis, 15,0-26,0 $\mathrm{cm}$ longa, panicula vulgo 5-ramosa, rachide et ramis atro-brunneis, minute tomentosulis squamosisque; bracteae maturae deciduae (non visae), bracteolae 1,0-2,0 $\mathrm{mm}$ longae, membranaceae, caducae; pedunculi ca. $1,0 \mathrm{~mm}$ longi. Receptaculum campanulatum, in pedunculo sessile, 2,0 mm longum, extus glabrum. Sepala 6, ovata, ciliata, $2,0 \mathrm{~mm}$ longa. Petala aequalia 5 , ovata, $5,0-12,0 \mathrm{~mm}$ longa, $4,0-7,0$ $\mathrm{mm}$ lata, suborbiculata $6,0-7,0 \mathrm{~mm}$ diam., ciliata, viridula. Androphorum album explanatum ca. $2,0 \mathrm{~cm}$ longum, $5,0-9,0 \mathrm{~mm}$ latum, staminibus ca. 70 fertilibus pauci-seriatis annulum basalem cingentibus, ad ligulam staminibus destitutum et ad galericulum multis staminibus cum filamentis brevibus instructum; ovarium 2-(3)-5-loculare, loculis multiovulatis; stylus brevissimus. Pyxidium lignosum, sessile, cylindricum vel campanulatum, ad basin obtuserotundatum, usque ad $10,0 \mathrm{~cm}$ longum, $8,0 \mathrm{~cm}$ diam.; zona calycaris usque ad $8,0 \mathrm{~cm}$ supra basim fructum cingens; vitta interzonalis 1,0-2,5 $\mathrm{cm}$ alta, convexa, margine superiore integra; zona superior spadicea, convexa, orbicularis usque ad 7,0 cm diam.; pericarpium 1,0-2,0 cm crassum. Operculum usque ad $2,0 \mathrm{~cm}$ altum, $7,0 \mathrm{~cm}$ diam., lignosum, laevigatum, superne fere planum in medio obsolete umbonatum, inferne concavum, subtus inaequaliter trisepta- 
tum. Semina pauci-numerosa, usque ad fere $5,0 \mathrm{~cm}$ longa, $2,0 \mathrm{~cm}$ crassa, angulato-ovata, triangularia, ad extremitates obtusa, nigrescentia papillosaque.

Corythophora rimosa W. Rodr. imprimis differt a $C$. alta Knuth trunci cortice profunde fisso, colore florum (petala viridula et androphorum album), fructibus maioribus latioribusque et dimensionibus seminum.

Habitat in Brasília, Amazonas, circa Manaus in silvis non inundabilibus, ubi satis frequens. "Castanha-jacaré", "castanha-casca-jacaré" vel "castanharana" incolis appelatur. Holotipus W. Rodrigues \& D. Coelho 7830 (fl. 11/5/1966), in Herb. INPA n. 17133.

\section{MATERIAL ADICIONAL EXAMINADO}

Amazonas. Manaus: estrada do Aleixo, $\mathrm{Km} \mathrm{13;} \mathrm{mata} \mathrm{de} \mathrm{terra} \mathrm{firme.} \mathrm{Árvore} \mathrm{bastante}$ grande; pétalas verdes herbáceas, androceu branco, "Castanharana". A. Ducke s/n (fl. 17-4-1932) RB 23887, R,P; ibid. Reserva Florestal Ducke: E. Ferreira 58-297 (fl. 12-6-1958) INPA 6502; W. Rodrigues 5284 (fl. 13-6-1963) INPA 13952, amostra de madeira INPA X-1925; W. Rodrigues \& A. Loureiro 5809 (fl. 8-5-1964) INPA 14521; W. Rodrigues \& Osmarino 6899 (fl. 14-4-1965) INPA 15450; W. Rodrigues \& Osmarino 6912 (fl. 23-4-1965) INPA 15463; W. Rodrigues \& D. Coelho 7721 (fl. 19-4-1966) INPA 17026; J. Elias \& Byron 473 (fl. 17-3-1969) INPA 27653; W. Rodrigues, L. Coelho, D. Coelho 8468 (fr. 19-3-1968) INPA 20994; J. Aluisio 32 (fl. 4-7-1968) INPA 21269; O. Pires 24 (st. 12-12-1969) INPA 27801; G. T. Prance, J. F. Ramos, L. G. Rarias, B. W. Albuquerque \& J. E. Paula 10417 (fl. 17-3-1969) INPA 26191, NY; ibid., estrada Mauá: G. T. Prance, L. F. CoeIho, K. Kubitzki, P. J. M. Maas 11639 (fl. 26-3-1971) INPA 29951, NY; ibid., estrada Manaus-Itacoatiara, Km 139: L. Coelho, A. Miranda $\mathrm{s} / \mathrm{n}$ (fl. 22-6-1972) INPA 36022, madeira INPA $\mathrm{X}-4957$.

Corythophora rimosa W. Rodr. diverge da espécie segưinte especialmente pela casca do tronco fortemente fissurada longitudinalmente, flores com pétalas verdes e andróforo branco e frutos bem maiores.
É uma espécie comum nas matas de terra firme das cercanias de Manaus, onde é conhecida vulgarmente pelos nomes de "castanha-jacaré", "castanha-casca-jacaré" e "castanharana".

2 - Corythophora alta Knuth, Engl. Pflanzenreich: 51. 1939.

Amazonas. Manaus: Reserva Florestal Ducke: E. Ferreira 58-298 (fl. 12-6-1958) INPA 6503, madeira INPA X-697; $W$. Rodrigues \& D. Coelho 7569 (fl. 16-3-1966) INPA 16872; W. Rodrigues \& D. Coelho, 7572 (fr. 16-3-1966) INPA 16875; W. Rodrigues \& D. Coelho 7689 (fr. 14-4-1966) INPA 16994; W. Rodrigues \& Osmarino 8218 (fr. 11-8-1966) INPA 17605; W. Rodrigues \& Osmarino 8328 (fr. 14-2-1967) INPA 19319; W. Rodrigues \& al. 8475 (fr. 8-3-1968) INPA 21124, madeira INPA X-3897; W. Rodrigues 8718 (fr. 26-2-1970) INPA 27892; W. Rodrigues \& Osmarino 7114 (fr. 9-6-1965) INPA 15776; J. Aluísio 241 (fl. 29-10-1968) INPA 23871; J. Aluísio 242 (fl. \& fr. 18-12-1968) INPA 23874; J. Aluisio 243 (fl. 18-12-1968) INPA 23874; J. Elias \& Byron 472 (fl. 17-3-1969) INPA 27652; G. T. Prance \& al. 9023 (fl. 12-12-1968) INPA 25822, NY; G. T. Prance \& al. 10418 (fl. \& fr. 17-3-1969) INPA 26192, NY; ibid., estrada Manaus-Itacoatiara: $\mathrm{Km}$ 69: W. Rodrigues \& J. Chagas, 1726 (fr. 8-9-1960) INPA 8089, madeira INPA X-725; ibid.: Km 70: W. Rodrigues 8673 (fl. 27-1-1970) INPA 27845; ibid.: Km 68: W. Rodrigues 8726 (fl. 5-3-1970) INPA 27900, madeira INPA X-4101; ibid.: Km 150: A. Loureiro \& al. $\mathrm{s} / \mathrm{n}$ (fl. 12-5-1972) INPA 35754, madeira INPA X-4787; A. Loureiro \& al. $\mathrm{s} / \mathrm{n}$ (fr. 17-5-1972) INPA 35811, madeira INPA X-4825; A. Loureiro \& al. s/n (fr. 14-5-1972) INPA 35796, madeira INPA X-4813; ibid.: $\mathrm{Km}$ 138: $O$. Pires \& J. Lima 155 (fr. 15-6-1972) INPA 35977, madeira INPA X-4930; ibid.: Km 139: L. Coelho \& A. Miranda $\mathrm{s} / \mathrm{n}$ (fr. 21-61972) INPA 36017, madeira INPA X-4950; ibid.: estrada Manaus-Caracaraí; Km 22: W. Rodrigues \& L. Coelho 1940 (fl. 22-11-1960) INPA 8312, madeira INPA X-831; ibid: $\mathrm{Km}$ 23: G. T. Prance \& al. 3831 (fl. 28-12-1966) INPA 19455, NY; ibid.: rio Urubu entre cachoeira Iracema e estrada ManausItacoatiara: G. T. Prance \& al. 5094 (fl. 8-6-1968) INPA 21854, NY. 


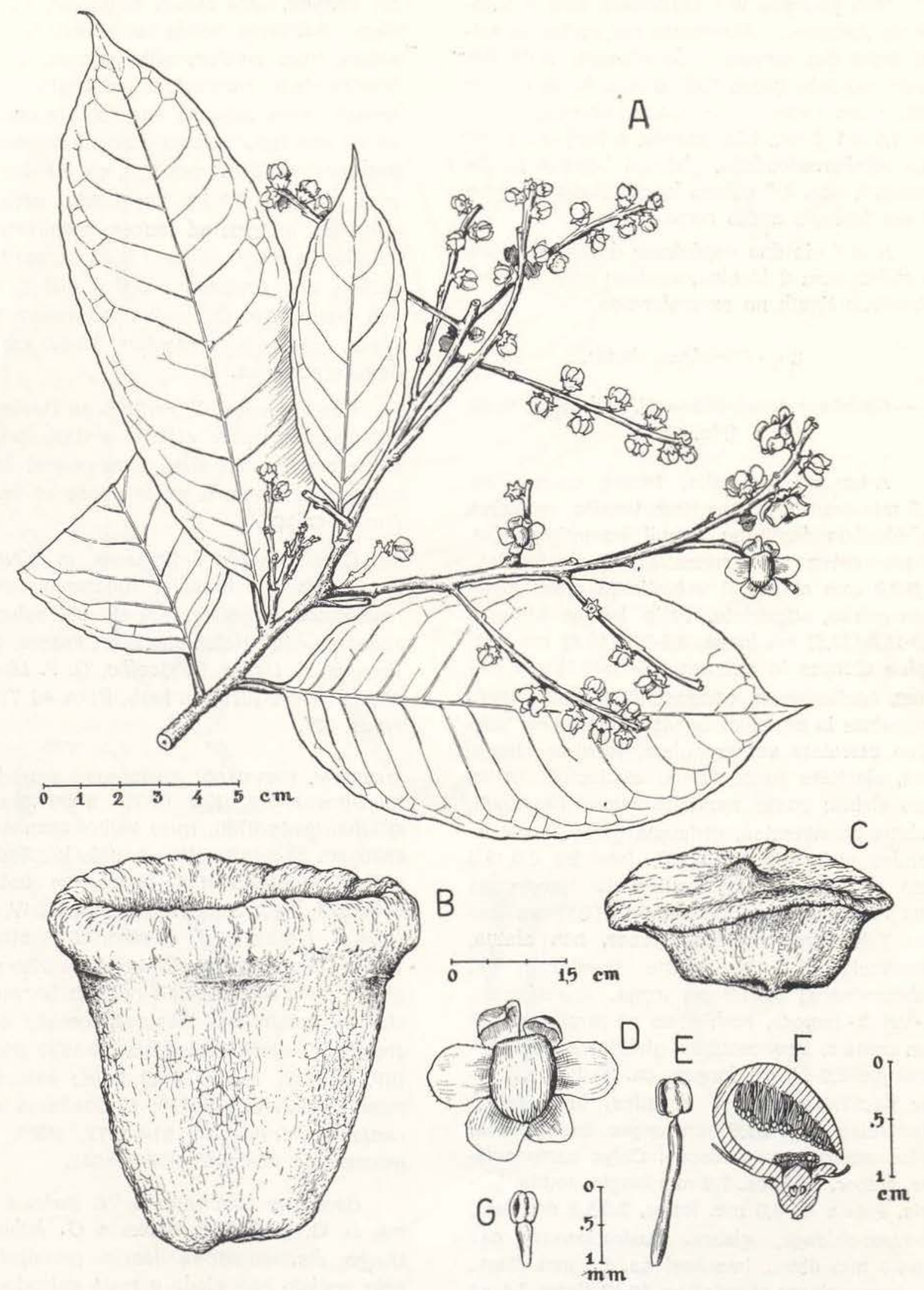

Fig. 1 Corythophora rimosa W. Rodrigues (W. Rodrigues \& D. Coelho 7830; O. Pires 24). A - Hábito de um ramo florífero. B - Fruto. G - Opérculo. D - Flor. E - Estame. F - Flor seccionada. G - Estame. (Desenhos de Júlio Dellome Filho). 
Pela primeira vez assinalada para o Esta. do do Amazonas. Abundante nas matas de terra firme das cercanias de Manaus, onde flo. resce durante quase todo o ano. As sementes não eram conhecidas: são fusiformes, cerca de $4,0 \times 1.0 \mathrm{~cm}$, triangulares, a face mais ampla semi-arredondada, glabras, lisas e pardoescuras, com 4-5 sulcos longitudinais imersos e um funículo muito curto.

A maioria dos espécimes de Manaus tem o ovário com 2 lóculos, nenhum com 3 como observou Knuth no exemplar-tipo.

\section{2 - Cariniana Casar.}

1 - Cariniana pauciramosa W. Rodrigues, n. sp. (Fig. 2)

Arbor ad $25 \mathrm{~m}$ alta, trunco cortice ca. $1,0 \mathrm{~mm}$ crasso, leviter fisso vestito, radicibus tabularibus destituta; ramuli hornotini glabri, nigrescentes vel brunneo-grisei, lenticellati. 1,0-2,0 mm crassi, ii vetustiores crassi, cortice griseo, substricto. Folia lamina elliptica $6,0-15,0-(23,5) \mathrm{cm}$ longa, $3,5-7,5(10,0) \mathrm{cm}$ lata, apice abrupte in acumen $0,5-1,8-(3,0) \mathrm{cm}$ longum, acutissimum contracta, ad basin breve angustata in petiolum breviter decurrens, margine serrulata vel crenulata, tenuiter chartacea, siccitate fusco-viridis, sublucida, utrimque glabra; costa nervique supra prominuli, subtus prominentes, utrimque glabri; nervi laterales utrimque 12-18-(25), inter se 2,0-18,0 $\mathrm{mm}$ remoti, venulis tenuissimis transversis non reticulatis; petiolus $1,5-4,0-(4,5) \mathrm{cm}$ longus, $1,0-2,0 \mathrm{~mm}$ crassus, glaber, non alatus, canaliculatus. Inflorescentia terminalis vel subterminalis, $0,5-1,0 \mathrm{~cm}$ longa, thyrsiformis, 2 -vel 3 -ramosa, rachidibus et ramis ca. 1,0 $\mathrm{mm}$ crassis, atrescentibus, glabris; spicae unaquaeque $2,0-4,0 \mathrm{~cm}$ longae, ca. 20 florae, leviter flactiflexae. Flores sessiles, lutescentes. Bracteolae ca. 1,0-1,5 mm longae, triangulares, apice acuto, mox caducae. Calyx campanulatus, glaber, lobis ca. $1,0 \mathrm{~mm}$ longis, acutis, glabris. Petala $4,0-8,0 \mathrm{~mm}$ longa, $2,0-5,0 \mathrm{~mm}$ lata, obovato-oblonga, glabra. Androphorum ca. 3,5-4,0 mm "diam., ima basi ca. $1,5 \mathrm{~mm}$ diam., ca. $3 \mathrm{~mm}$ altum, staminibus $10-12$ (intra 3-4 et ad apicem 7-9 affixis); antherae reflexae. Stylus minutus. Pyxidium sine operculum $6,5-10,0$ $\mathrm{cm}$ longum, infra zonam calycarem $2,7-3,0 \mathrm{~cm}$ diam., vasiforme, solide coriaceum, leviter curvatum, infra medium subtrigonum, ad vittam interzonalem contractum, ad basin obtusum, sessile; zona calycina linearis, vix conspicua, 2,0-4,0 mm infra apicem fructum cingens; vitta interzonalis $2,0-4,0 \mathrm{~mm}$ alta, ca. $2,5 \mathrm{~cm}$ diam.; zona superior 2,7-3,2 cm diam., reflexa, tenuissima, integra, ad faucem manifeste triangularis; pericarpium ca. $1,0 \mathrm{~mm}$ crassum, nec fissum nec costatum, cortice glabro, crustaceo instructum. Operculum convexum $2,3 \mathrm{~cm}$ diam., columella triangulari $5,0-6,0 \mathrm{~cm}$ longa. Semina non visa.

Haec species C. multiflorae Ducke et $C$. kuhlmanniae Ducke affinis, distinguitur praecipue petiolo non alato tenuioreque, inflorescentia satis minora, pauciramosa et fructuum dimensionibus.

Crescit valde infrequens in silvis non inundabilibus in Brasilia, Amazonas, via Manaus-Itacoatiara super $\mathrm{Km} 69$, ubi solummodo adhuc cognita. Holotypus G. T. Prance, W. Rodrigues, E. Lleras, D. Coelho, O. P. Monteiro, 17516 (fl. 5-9-1973) in herb. INPA 40.712, Isotypus, NY.

MATERIAL ESTUdAdO: Amazonas: estrada Manaus-Itacoatiara, $\mathrm{Km}$ 69-70, margem direita: W. Rodrigues 8790 , fruto velho apanhado do chão em 25-3-1970, INPA 27970; W. Rodrigues \& O.P. Monteiro 9114, flores em botão em 21-8-1973, INPA 39589; G. T. Prance, W. Rodrigues, E. Lleras, D. F. Coelho, O. P. Monteiro, 17516 , forest on terra firme. Tree $25 \mathrm{~m} \times 40 \mathrm{~cm}$ diam.. Bark brown slash reddish brown, with shallow longitudinal fissures; corolla and androphore yellow. Flores em 5-9-1973 (Holotipo INPA 40712, isotipo NY); ibid.: estrada Manaus-Itacoatiara, $\mathrm{Km}$ 139: L. Coelho \& A. Miranda $\mathrm{s} / \mathrm{n}$, frutos em 21-4-1972, INPA 36013. amostra de madeira INPA x-4947.

Cariniana pauciramosa W. Rodr. é próxima de $C$. multiflora Ducke e C. kuhlmannil Ducke, distinguindo-se destas principalmente pelo pecíolo não alado e mais delgado, inflorescência bem menor e muito pouco ramificada e pelas dimensóes do fruto. 


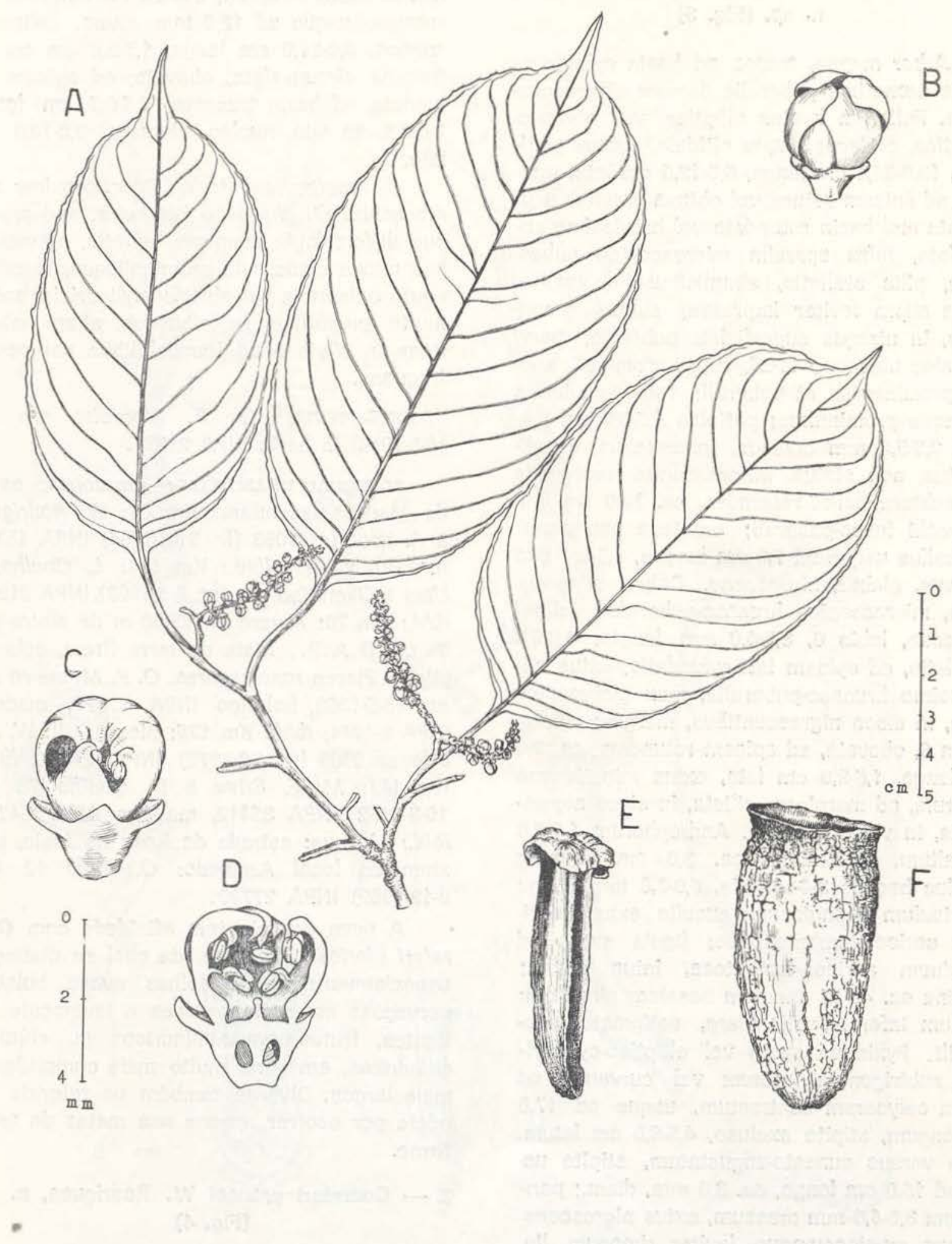

Fig. 2 Cariniana pauciramosa W. Rodrigues (G. T. Prance \& al. 17516; W. Rodrigues 8790). A - Hábito de um ramo florífero. B - Flor. C - Flor mostrando o andróforo. D - Flor seccionada longitudinalmente E - Opérculo. F - Fruto. (Desenhos de Júlio Dellome Filho) 


\section{3 - Couratari Aubl.}

\section{1 - Couratari longipedicellata W. Rodrigues, n. sp. (Fig. 3)}

Arbor magna, trunco ad basin cylindrico, ramis hornotinis puberulis demum glabrescentibus. Foliorum lamina elliptica vel obovatoelliptica, coriacea, supra nitidula, subtus pallidior, $13,0-24,0 \mathrm{~cm}$ longa, $5,0-12,0 \mathrm{~cm}$ lata, integra, ad apicem retusa vel obtusa breviter acuminata and basin rotundata vel brevissime angusdata, infra sparsim microscopice pubescens, pilis stellatis, simplicibusque vestita; costa supra leviter impressa, subtus prominens, in utraque superficies puberula; nervi laterales utrimque 11-20, supra prominuli, subtus prominentes et puberuli; rete venularum utrimque prominulum; petiolus $1,5-2,0 \mathrm{~cm}$ longus, 2,0-5,0 mm crassus, tomentellus, canaliculatus, non alatus. Inflorescentia terminalis vel subterminalis, racemosa, ca. $20,0 \mathrm{~cm}$ longa, rachi fusco-puberuli; bracteae non visae; pedicellus usque ad $7,0 \mathrm{~cm}$ longus, $1,0-2,0 \mathrm{~mm}$ crassus, glaber, nigrescens. Calyx campanulatus, microscopice brunneo-puberulus vel ni grescens, lobis $6,3,0-4,0 \mathrm{~mm}$ longis, 4,0-6,0 $\mathrm{mm}$ latis, ad apicem late-rotundatis, extus minutissime brunneo-puberulis, mox glabrescentibus, in sicco nigrescentibus, margine ciliata. Petala 6, obovata, ad apicem rotundata, ca. 3,0 $\mathrm{cm}$ longa, 1,5-2,0 cm lata, extus minutissime puberula, ad marginem ciliata, in sicco nigrescentia, in vivo purpurea. Androphorum 1,8-2,0 $\mathrm{cm}$ altum, explanatum ca. $3,0 \mathrm{~cm}$ longum; annulus basalis $3-4$-serialis, $5,0-7,0 \mathrm{~mm}$ diam.; galericulum spongiosum, cucullo extus laevigato sericeo-tomentosoque; ligula extus ad marginem sericeo-tomentosa, intus glabra; stamina ca. 40-45 annulum basalem cingentia; ovarium inferum, triloculare, estigmate subsessili. Pyxidium ovato vel elliptico-cylindricum, subtrigonum, rectum vel curvatum, ad zonam calycarem contractum, usque ad 17,0 $\mathrm{cm}$ longum, stipite excluso, 4,5-6,0 $\mathrm{cm}$ latum, basin versus cuneato-angustatum, stipite us que ad 15,0 cm longo, ca. 3,0 mm, diam.; pericarpium $3,0-4,0 \mathrm{~mm}$ crassum, extus nigrescens glabrum cruštaceumque, leviter rimosum, lig nosum; zona calycaris linearis, protrusula, 4,0-20,0 mm infra apicem cincta; vitta interzonalis subtrigona, erecta, ca, $4,0 \mathrm{~cm}$ diam.; zo- na superior subtrigona, reflexa vel erecta, ad faucem 3,5-4,0 cm diam.; operculum convexum. non radiatim striatum, centro vix umbilicatum, rubrum, usque ad $12,0 \mathrm{~mm}$ altum, columella trigona, $9,0-11,0 \mathrm{~cm}$ longa, $1,5-2,0 \mathrm{~cm}$ crassa. Semina circum-alata, obovata, ad apicem rotundata, ad basin truncata, 3,5-9,5 cm longa, 2,0-2,5 cm lata, nucleo obovato, $8,0-10,0 \mathrm{~mm}$ lato.

C. longipedicellata W. Rodr. proxime affinis habitu C. gloriosae Sandwith. sed praecipue differt foliis nunquam bullatis, nervationibus minus numerosis prominulisque, fructibus ovato-cylindricis vel elliptico-cylindricis, saepe multo longioribus latioribusque, etiam habitatione in silvis haud inundabilibus ubi parum frequens.

HolotyPUS: $O$. P. Monteiro $\mathrm{s} / \mathrm{n}$ (fl. 18-5-1969) in herb. INPA 27371.

MATERIAL EXAMINADO: Amazonas: estrada Manaus-Itacoatiara: $\mathrm{Km}$ 66: W. Rodrigues \& A. Loureiro 7063 (fr. 31-8-1965) INPA 15725. madeira x-3227; ibid.: $\mathrm{Km}$ 104: L. Coelho, J. Lima \& Osmarino s/n (st. 8-5-1968) INPA 21222; ibid.: $\mathrm{Km}$ 70: Árvore de $25-30 \mathrm{~m}$ de altura por $35 \mathrm{~cm}$ D.A.P. . Mata de terra firme, solo argiloso. Flores roxo-escuras. O. P. Monteiro $\mathrm{s} / \mathrm{n}$ em 18-5-1969, holotipo INPA 27371, madeira INPA x-4044; ibid.: Km 125: picada XIII: W. Rodrigues 8689 (fr. 4-2-1970) INPA 27861; ibid.: $\mathrm{Km}$ 143: M. F. Silva \& D. Coelho 78 (fr. 10-3-1972 INPA 35412, madeira INPA x-4762; ibid.: Manaus: estrada do Rosa de Maio, próximo do local Armando: O. Pires 12 (fr. 9-12-1969) INPA 27799.

A nova espécie tem afinidade com Couratari gloriosa Sandwith, da qual se distingue especialmente pelas folhas nunca bulatas, nervações menos numerosas e levemente salientes, frutos ovado-cilíndricos ou elípticocilíndricos, em geral muito mais compridos e mais largos. Diverge também da referida espécie por ocorrer apenas nas matas de terra firme.

2 - Couratari prancei W. Rodrigues, n. sp. (Fig. 4)

Arbor ad $30 \mathrm{~m}$ alta, rami hornotini pube. ruli, vetustiores glabri. Foliorum lamina elliptica vel obovata, chartacea, $6,0-12,0 \mathrm{~cm}$ longa, 


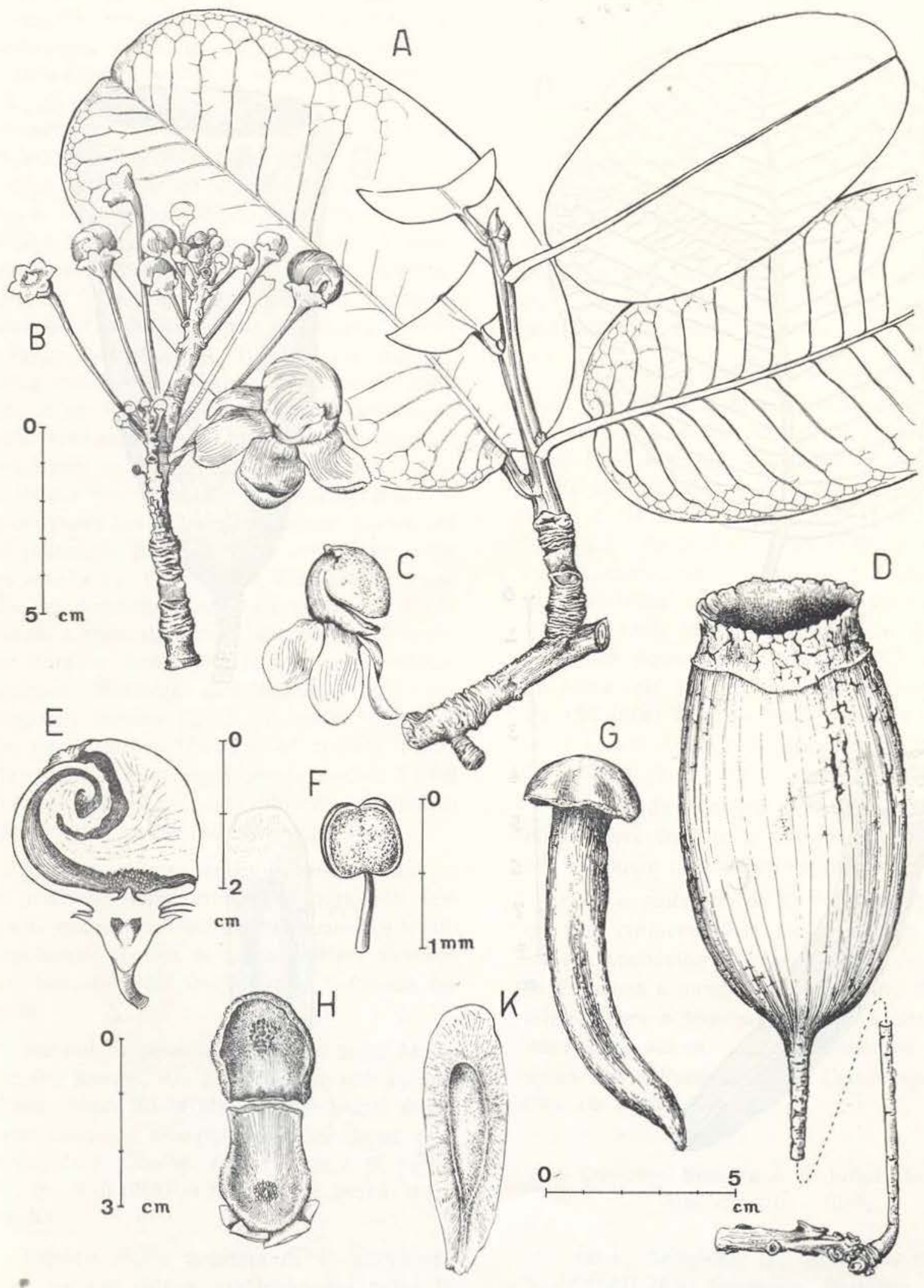

Fig. 3 Couratari Iongipedicellata W. Rodrigues (O. P. Monteiro s/n INPA 27371; W. Rodrigues \& A. Loureiro 7063). A - Hábito de um raminho estéril. B - Inflorescência. C - Flor vista de perfil. D - Fruto. E - Flor seccionada. F - Estame. G - Opérculo. H - Andróforo estendido mostrando a face interna. K - Semente (Desenhos de Júlio Dellome Filho). 

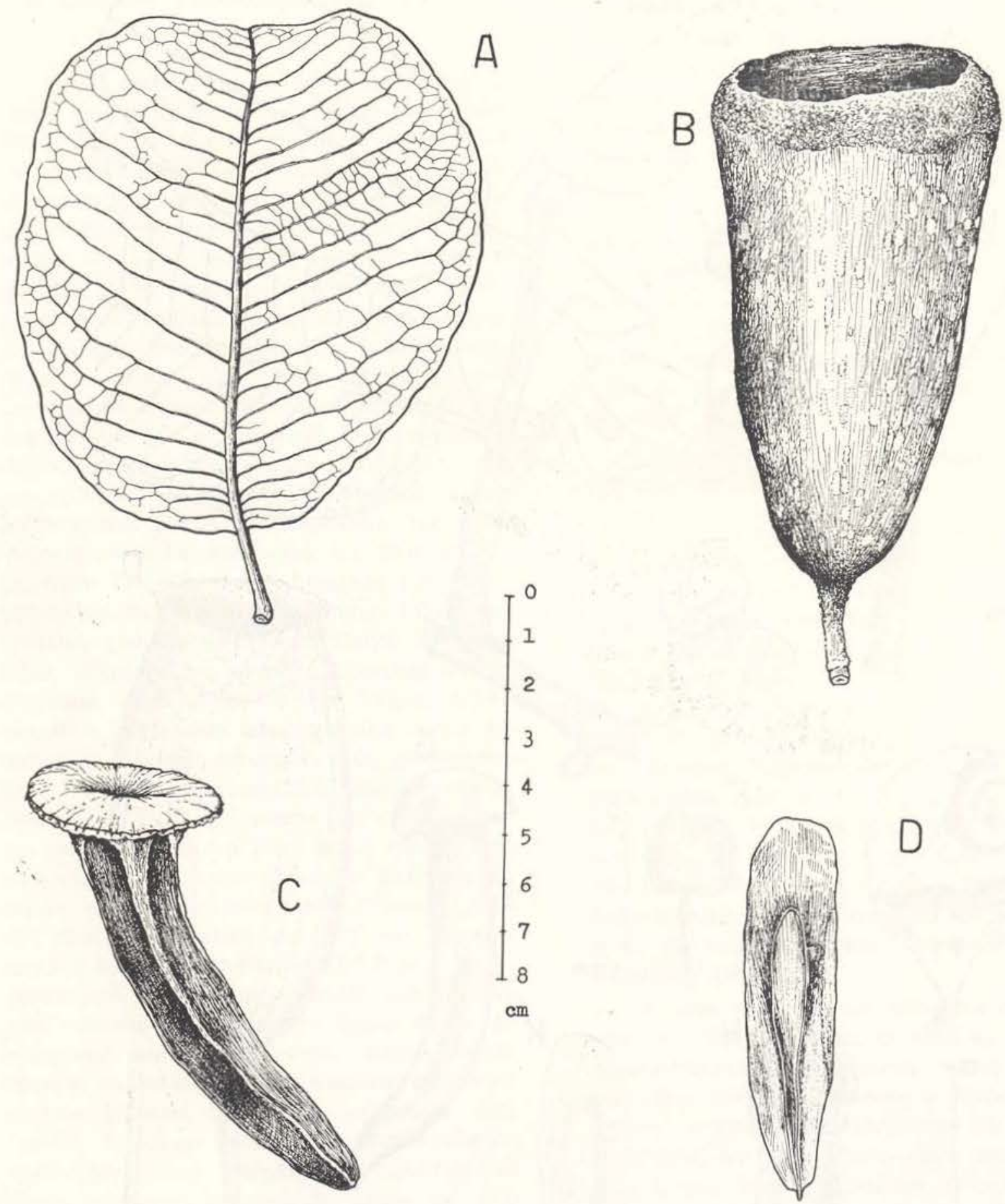

Fig. 4 Couratari prancei W. Rodrigues (G. T. Prance \& al. 7755), A - Folha. B - Fruto. C - Opérculo. D Semente. (Desenhos de Júlio Dellome Filho). 
4,0-9,0 cm lata, margine integra vel undulata, ad apicem obtuse-emarginata, rotundata vel obtuse-acuta, ad basin rotundata, subtus pallide denseque arachnoideo-stellato-puberula ut in $C$. guianensis Aubl.; costa supra plana vel prominula et tomentella, subtus prominens pu berulaque; nervi 13-24-jugi, supra plani et ii juniores puberuli, infra prominentes puberulesque: rete venularum utrimque prominulum; petiolus $8,0-20,0 \mathrm{~mm}$ longus, $1,0-2,0 \mathrm{~mm}$ latus, puberulus, canaliculatus, non alatus. Inflorescentia et flores non visi. Pyxidium obconicecylindricum sursum versus dilatatum, leviter curvatum, subtrigonum, $11,0-14,0 \mathrm{~cm}$ longum, 4,5-5,3 cm maxime diametro, ad basin obtusum; stipe 15,0-18,0 mm longus; pericarpium extus lenticellis albis, magnis conspicuisque 2,0-6,0 $\mathrm{mm}$ longis indutum, solide coriaceum, ca. 2,0-5,0 $\mathrm{mm}$ crassum; zona calycaris ca. $9,0 \mathrm{~cm}$ supra basim fructum cingens, planis vel non protrusis, plus minusve irregularis; vitta interzonalis ca $1,5 \mathrm{~cm}$ alta, convexa, non sulcata vel porcata; zona superior $4 \mathrm{~cm}$ diam. inflexa, circularis, tenui, lacerata et irregulariter dentata; operculum inconspicue radiatim sulcatum, distincte umbillicatum, columella triangulari. Semina $7,0-9,0 \mathrm{~cm}$ longa, $1,7-2,3 \mathrm{~cm}$ lata, circum-alata, obovata, ad apicem rotundata, ad basin truncata, brunnea, nucleo 5,0-6,0 $\mathrm{cm}$ longo, 6,0-9,0 $\mathrm{mm}$ lato, obovato, ad apicem obtuso, basin versus sensim angustato.

Maxime affinis est $C$. guianensi Aubl., a qua imprimis foliis minoribus, pericarpii lenticellis maioribus, fructus zona superiore tenui, irregulariter dentata et inflexa differt. Species nova haec botanici Dr. Ghillean T. Prance dedicata.

Habitat in Brasilia, Acre, via Sena Madureira-Rio Branco, $\mathrm{Km} \mathrm{3-6,} \mathrm{in} \mathrm{silvis} \mathrm{non} \mathrm{inunda-}$ bilibus. Arbor $30 \mathrm{~m}$ alta, $80 \mathrm{~cm}$ trunci diam. fructu brunneo, albo-punctato. Holotypus G.T. Prance, D. F. Coelho, J. T. Ramos \& G. Farias 7755 (fr. 2-10-1968) in herb. INPA 24553, isotypus NY.

Espécie muito próxima de $C$. guianensis Auble, da qual difere principalmente pelas foIhas menores, pericarpo com lenticelas maiores, zona superior do fruto fina, irregularmente dentada e convexa.
3 - Couratari multiflora (J. E. Smith) Eyma, Polygon., Guttif, Lecyth. Surinam: 60. 1932

(Fig. 5)

BRASIL: Pará: Belém, IPEAN, Reserva Aurá: J. M. Pires \& N. T. Silva 11887 (fl. 12-7-1968) IAN. Amazonas: Manaus, Reserva Florestal Ducke: W. Rodrigues \& A. Loureiro 5901 (fl. 11-6-1964) INPA; ibid:: M. Freitas, D. Coelho, L. Coelho 12 (fr. 5-12-1969) INPA; Estrada Manaus-Itacoatiara; $\mathrm{Km}$ 68: $W$. Rodrigues 8796 (fl. 14-4-1970) INPA; ibid.: Km 152: W. Rodrigues 9092 (fl. 6-6-1973) INPA.

venezuela: Território Federal Delta Amacuro: Leste de Rio Grande: Leste Noroeste de El Palmar, cerca dos limites do Estado de Bolivar: L. M. Berti 226 (fr. 29-5-1964) VEN.. Estado de Bolivar: Leste de El Palmar, estrada La Tigra: L. M. Berti 314 (estéril 12-15-Jul.-1964) VEN. N.E. de Upata ( $\pm 62^{\circ} 13^{\prime} W ; 8^{\circ} 16^{\prime} \mathrm{N}$ ), El Paraiso camp., alt. $\pm 500 \mathrm{~m}:$ F. J. Breteler 5070 (st. 20-3-1966) VEN; ibid.: F. J. Breteler 5073 (st. 20-3-1966) VEN. - Altiplanície de Nuria, Quebrada Agua Linda, 8-11 Km "E". de Hato de Nuria, alt. $550 \mathrm{~m}$ : J. A. Steyermark 86496 (fr. 19-7-1960) VEN. - Rio Toro entre la Reforma e Puerto Rico: J. A. Steyermark 88100 (st. 15-12-1960) VEN.

Árvore da mata de terra firme facilmente distinguivel durante a floração. Despe-se de sua folhagem para cobrir-se de flores róseas.

Sob o sinônimo de C. fagifolia (Miq.) Eyma e C. coriacea Mart. ex Berg., esta espécie só era conhecida do Suriname, Guiana, Guiana Francesa e parte oriental da Amazônia brasileira (Pará e Maranhão). Com a citação dos espécimes acima, sua dispersão se estende agora até a Venezuela e o Estado do Amazonas, no Brasil.

4 - Couratari Stellata A. C. Smith, Am. Journ. Bot. 26:410. 1939 .

PARÁ: Belterra: G. Black 47-1907 (fl. 31-10-1947) IAN; Santarém, Planalto: Cachoeira de Curuauna: R. L. Froes 31105 (fl. fr. out.1954) IAN; ibid.: R. L. Froes 31105-B (fr. 15-1-1954) IAN; ibid.: Taperinha, paraná de Itu- 


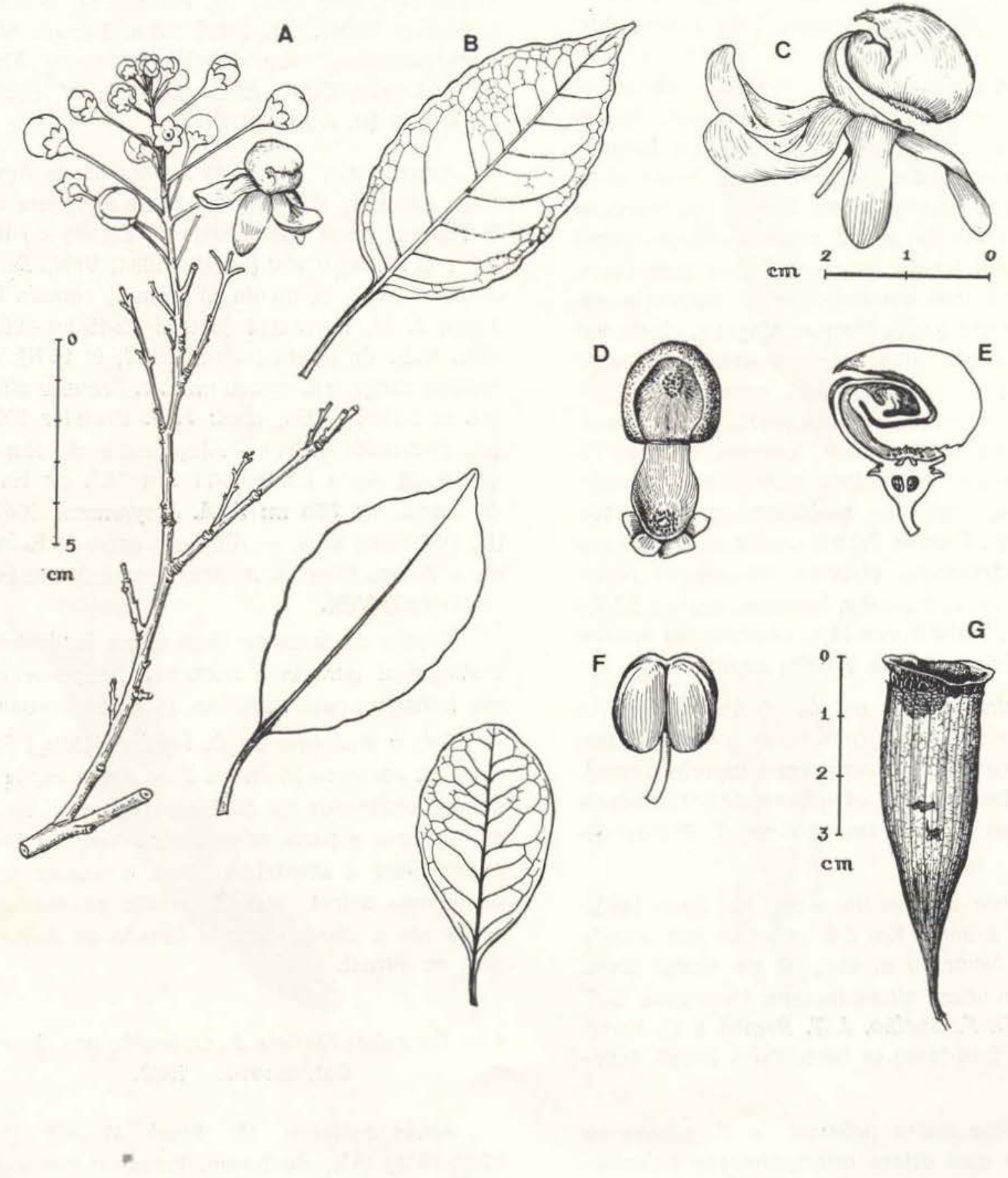

Fif. 5 Couratari multiflora (J. E. Smith) Eyma (M. Freitas \& al. 12; W. Rodrigues 8796). A - Ramo florifero. B - Folhas. $\mathbf{C}$ - Flor. D - Andróforo estendido mostrando a face interna. E - Flor seccionada. F - An. tera. F - Fruto. (Desenhos de Júlio Dellome Filho). 


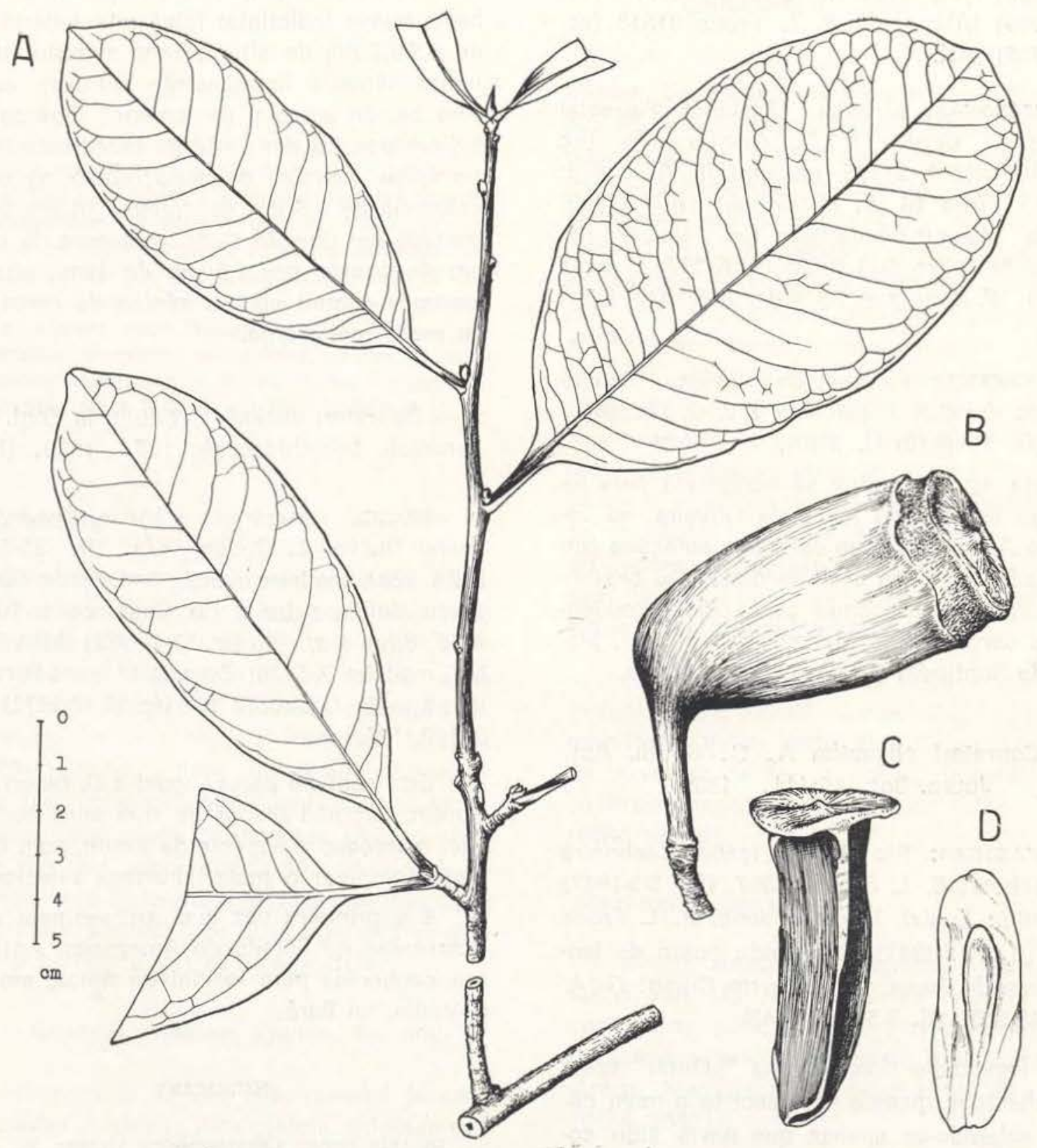

Fig. 6. Couratari duckei R. Knuth (M. F. Silva \& al. 783). A - Raminho estéril. B - Fruto. C - Opérculo. D - Semente. (Desenhos de Júlio Dellome Filho). 
qui: R. L. Froes 31200 (st. 3-12-1954) IAN; ibid.: Santa Rosa: R. L. Froes 31465 (fr. fev.-1955) IAN; ibid.: R. L. Froes 31470 (fl. \& fr. fev.-1955) IAN; ibid.: R. L. Froes 31479 (fr. fev.-1955); ibid.: R. L. Froes 31497 (fr. 10-1-1955) IAN; Planalto de Santarém: R. L. Froes 31506 (st. 10-1-1955) IAN; ibid.: R. L. Froes 31517 (st. 10-1-1955) IAN; ibid.: R. L. Froes 31518 (st. 10-1-1955) IAN.

AMAZONAS: Manaus: Reserva Florestal Ducke: L. Coelho \& D. Coelho $\mathrm{s} / \mathrm{n}$ (st. 14-3-1968) INPA 21175, madeira INPA X-3873; ibid.: O. Pires 14 (fl. 11-12-1969) INPA 27803; Estrada Manaus-Itacoatiara: $\mathrm{Km}$ 145: W. Rodrigues 8610 (fr. 5-11-1969) INPA 27717; ibid.: Km 125: W. Rodrigues 8874 (fr. 19-5-1970) INPA 28238.

SURINAME: montes de Nassau: campo perto do $\mathrm{Km} \mathrm{5,9:} \mathrm{J.} \mathrm{Lanjouw} \mathrm{\&} \mathrm{J.} \mathrm{C.} \mathrm{Lindeman}$ 2419 (fr. 3-3-1949) U, INPA.

Esta espécie antes só conhecida pela localidade típica, São Paulo de Oliveira, no Estado do Amazonas, com as novas coleções acima citadas tem sua área de dispersão bastante ampliada, aparecendo com certa freqüência nas cercanias de Manaus (Amazonas), planalto de Santarém (Pará) e em Suriname.

5 - Couratari oligantha A. C. Smith, Am. Journ. Bot. 26:411. 1939

AMAzonas: Rio Negro: Içana, Cachoeira Malacacheta: R. L. Froes 22307 (fr. 5-5-1947) IAN; ibid.: Enuixi, lago Dondona: R. L. Froes 22372 (fl. 18-5-1947) IAN; ibid.: posto de Içana, acima de Tunuí, beira do rio Cuiari: G. A. Black 48-2631 (fl. 7-5-1948) IAN.

A localidade típica e seu "habitat" eram desconhecidos quando foi descrita a nova espécie, sabendo-se apenas que havia sido coIhida no alto rio Negro. Pelas novas coleçōes acima citadas esses dados podem ser compiementados. Seu ambiente natural é a beira de rio ou igapó.

Seus frutos também ainda não eram conhecidos. São um tanto semelhantes aos de
C. tenuicarpa A. C. Smith e C. riparia Sandwith, apresentando a seguinte forma: ca. 4,5 $\mathrm{cm}$ de comprimento por $3,5 \mathrm{~cm}$ de diâmetro na extremidade superior, coniforme, estreitado em direção à base; pericarpo pardo escuro, todo estriado longitudinalmente, coriáceo e duro; zona calícina ca. de $4,0 \mathrm{~cm}$ acima da base, quase indistinta; faixa interzonaria ereta de $0,5-0,7 \mathrm{~cm}$ de altura; zona superior inteira, muito tênue a ligeiramente inflexa; estípite fino ca. de $2,5 \mathrm{~cm}$ de compr.; opérculo de $1,5 \mathrm{~cm}$ por $1,0 \mathrm{~cm}$ de larg., levemente imerso no ápice com um pequeno relevo no centro; columela de $3,5 \mathrm{~cm}$ de compr., triangular, estreitado em direção distal; semente de ca. 3,5 $\mathrm{cm}$ de compr. por $1,5 \mathrm{~cm}$ de larg., obovada, castanho-escura com o núcleo de ca. $1,0 \mathrm{~cm}$ na parte mais larga.

6 - Couratari duckei R. Knuth, in Engl. Pflanzenreich, Lecythidaceae: 135. 1939. (Fig. 6)

BRASIL. Amazonas: Manaus, Reserva Florestal Ducke: L. Coelho s/n. (fr. 25-3-1957) INPA 5204, madeira X-609; - Estrada ManausPorto Velho, entre o rio Castanho e Tupana: M. F. Silva \& al. 783 (fr. 17-7-1972) INPA 36874, NY, madeira X-5128; Estrada Manaus-Porto VeIho, Km 85: L. Coelho 126 (fr. 20-12-1971) INPA 35132, NY.

Esta espécie parece igual à $C$. tauari Berg. porém, por não dispor do tipo para comparação, mantenho a espécie de Knuth, com o qual pude comparar o material acima relacionado.

É a primeira vez que se assinala a sua ocorrência no Estado do Amazonas. Antes só era conhecida pela localidade típica, em Juriti Velho, no Pará.

\section{SUMMARY}

In this paper Corythophora rimosa W. Rodr. Cariniana pauciramosa W. Rodr., Couratari longipedicellata W. Rodr. and Couratari prancei W. Rodr. are described as new and Corythophora alta $R$. Knuth, Couratari multiflora (J. E. Smith) Eyma, Couratari stellata A. C. Smith, C. oligantha Sandwith and C. duckei R. Knuth are discussed here as little known Lecythidaceae from Amazonia. 